\title{
Study on The Method of Channel Siltation Recognition Based on SVM
}

\author{
Xun Zhang ${ }^{1}$ Wenhai $\mathrm{Xu}^{2}$ Lili Dong $^{1}$ Wenyan Tang ${ }^{1}$ \\ ${ }^{1}$ Department of Automatic Testing and Control, Harbin Institute of Technology, Harbin, 150001, P. R. China \\ ${ }^{2}$ College of Information Engineering, Dalian Maritime University, Dalian, 116026, P. R. China
}

\begin{abstract}
A method to recognize the channel siltation using support vector machine (SVM) is developed. The method is based on the system that the transducer arrays are laid out respectively on each side of the channel. Through the analysis of sonar signal acquired by data acquisition unit, the features to recognize the siltation are extracted. By recognizing verification of the siltation in one channel, the results show that the method can recognize the channel siltaion correctly. Compare with the traditional approaches of ANN, the method has the advantages of high recognition precision $(>90 \%)$.
\end{abstract}

Keywords: Channel siltation, Transducer arrays, Support vector machine (SVM)

\section{Introduction}

Siltation in the channel exists universally and it can affect navigation safety and benefit. At present, there are mainly two kinds of method to monitor the sediment in the channel. One is the method of shipborne devices, that is, applying shipborne devices such as multibeam sonar and side scan sonar etc. to carry out on-site measurement. However, this method needs the survey vessel to measure in the channel repeatedly, and must dynamically monitor the change of the sediment situation in the channel, so it will disturb the channel operation and need to monitor the whole channel. And the workload is large and the cost is high, especially the method can't carry out real-time monitoring [1]-[2]. The other method is based on numerical forecast model: according to many accumulated sediment data and combined with the fundamental theories of tide, weather, ocean current, hydromechanics and hydrodynamics, some mathematical models are set up to predict the rules of sediment in the channel [3]-[4]. Apparently, the method has a certain limitation in use and the situation of the given harbor must be known clearly, so the method can't be used widely. In addition, acoustic channel is complex, time varied and space varied.
Especially in alluvial channel, the attenuation of the sonar signal is great, the signal-to-noise ratio is lower and the sample can't be acquired easily. Thus, in this paper a recognition method of siltation using SVM was developed. Based on the method, a system of monitoring channel siltation situation in real time was constructed. Through the experiment of recognizing the siltation in one channel, the recognition method of siltation was proved to be practical.

\section{Support vector machine}

SVMs have become an increasingly popular nonparametric methodology for developing classification models. In their basic form, SVM learn linear decision rules of the following form:

$$
D(x)=\operatorname{sign}(w \cdot x+b)
$$

The decision rule $\mathrm{D}(\mathrm{x})$ is equal to +1 for positive values of $(w \cdot x)+b . D(x)$ is equal to -1 otherwise. The weight vector $\mathrm{w}$ and the threshold $\mathrm{b}$ are found during training and they describe a hyperplane. Observations (x) are classified according to the side of the hyperplane in which they are located. During training, the SVM approach calculates the hyperplane that classifies most training samples correctly while keeping the largest possible "margin" around the hyperplane. If all the training samples can be classified without error, the "margin" is the distance from the hyperplane to the closest training samples. For cases in which the data can not be correctly classified by a linear decision rule, the training samples $\mathrm{x}$ are mapped (by a function $\Phi(x)$ ) to a feature space where the data become linearly separable. The resulting decision rule has the form:

$$
D(x)=\operatorname{sign}(w \cdot \Phi(x)+b)
$$

Again, $w$ and $b$ are found during training. Because $\mathrm{w}$ lies in the feature space, it is common practice to solve a dual optimization problem to find the $\mathrm{w}$ that maximizes the margin. This leads to the following nonlinear decision function:

$$
D(x)=\operatorname{sign}\left(\sum_{\forall x_{i} \in S} a_{i} \lambda_{i}\left(\Phi\left(x_{i}\right) \cdot \Phi(x)\right)+b\right)
$$




$$
D(x)=\operatorname{sign}\left(\sum_{\forall x_{i} \in S} \alpha_{i} K\left(x_{i}, x\right)+b\right)
$$

Where $K\left(x_{i}, x\right)$ is a kernel function (for example, for a linear kernel, $K\left(x_{i}, x\right)=\left(x \cdot x_{i}\right)$ and for a radial basis function kernel, $\left.K\left(x_{i}, x\right)=\exp \left(-\gamma\left\|x \cdot x_{i}\right\|^{2}, \gamma>0\right)\right)$ of a pattern to be classified $\mathrm{x}$ and a training pattern $x_{i} . \mathrm{S}$ is a subset of the training set, $\lambda_{i} \in(-1,1)$ is the label of pattern $X_{i}, \alpha_{i}$ is the Lagrange multiplier associated with the pattern $x_{i}$, and $\mathrm{b}$ is the threshold. As described in [5]-[6], during training, optimization of $\alpha_{i} \geq 0$ is achieved by:

$$
\min _{\alpha}\left(\alpha^{T} \Lambda K \Lambda \alpha+C \sum_{j} \varepsilon_{j}\right)
$$

constrained by $\lambda_{i} D(x) \geq 1-\varepsilon_{j}, \forall x_{j}$ in the training set $\Lambda$ is a diagonal matrix containing the labels $\lambda_{j}$, and the matrix $\mathrm{K}$ stores the values of the kernel function $K\left(x_{i}, x\right)$ for all pairs of training patterns. $\varepsilon_{j}$ is slack variables which allow for class overlap, controlled by the penalty weight $\mathrm{C}>0$. For $\mathrm{C} \rightarrow \infty$, no overlap is allowed. During optimization, the values of all $\alpha_{i}$ become 0 , except for the $\alpha_{i}$ associated with the support vectors (a special subset of the training samples). Consequently the support vectors are the only patterns needed in deciding the position of the decision boundary. Summarizing the SVM approach, the input vector $\mathrm{x}$ and the support vectors $X_{i}$ are mapped into a feature space where the necessary dot products are computed by using the kernel function K. The kernel function is chosen a priori, and it determines the type of classifier (linear or radial basis function). The penalty weight $\mathrm{C}$ is also chosen a priori, and it controls the amount of allowed overlap between classes. All other parameters (the number of support vectors and weights and the threshold) are found during training [7].

\section{Sonar signal acquisition and feature extraction}

\subsection{Construction of experiment system}

A siltation monitoring experiment system was constructed in one China's eastern coastal silted harbor (channel depth is $12 \mathrm{~m}$, width $200 \mathrm{~m}$ ). One emission transducer array is set up in one side of the channel and a receiving one is set up in the other side emission both $10 \mathrm{~cm}$, then the measuring resolution is $10 \mathrm{~cm}$ too.
If the measuring thickness of the sludge is $1 \mathrm{~m}$, the transducer number $\mathrm{N}$ is 10 . In order to effectively contribute to the safe navigation, the transducer arrays should set up aslant and the horizontal distance between two contiguous transducers is $10 \mathrm{~cm}$. At one

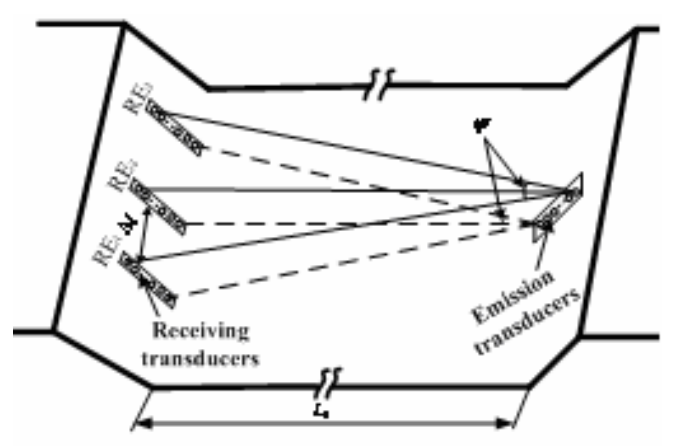

Fig.1: Schematic of the arrangement of the transducer arrays ( $\Delta l$ : Distance between two receiving array; $\psi$ : Horizontal cover sector angular of the ultrasonic; $L_{0}$ : Channel width;).

site, in order to monitor the siltation situation of a larger area, one emission array and several receiving arrays need to be adopted. Fig. 1 is the scheme of the arrangement of the transducer arrays in the channel. The horizontal cover sector angular $\psi$ depends on the transmitting power of the emission units. The number of the receiving array $\mathrm{M}$ depends upon the sector angular $\psi$ and the distance $\Delta l$ between two receiving arrays. The calculation formula is given as:

$$
M=\frac{2 \cdot L \cdot \tan (\Psi / 2)}{\Delta l}+1
$$

For the channel width $L_{0}$ is $200 \mathrm{~m}$, the longitudinal beam angle is $3^{\circ}$ and the transverse beam angle is $30^{\circ}$. Then the horizontal cover sector angular $\psi$ is $30^{\circ}$ too. According to the covered range of the sonar signal, the distance $\Delta l$ between two contiguous receiving arrays can be confirmed: $\Delta l=53 \mathrm{~m}$, the number of the receiving arrays is 3 . And the emission array consist of 10 units is set up on one side slope of the channel. The down-top number is $T R_{1}, T R_{2}, \ldots, T R_{10}$ in turn. On the other side, 3 receiving arrays are set up, from the left to the right, the number is $R E_{1}, R E_{2}, R E_{3}$, and each has 10 receiving units, the down-top number is $R E_{1,1}, R E_{1,2}, \ldots, R E_{1,10} ; R E_{2,1}, R E_{2,2}, \ldots$, $R E_{2,10}$ in turn. From the layout of the transducer arrays we can see that the distance between $T R_{i}$ and $R E_{2, i}$ is $L_{0}+0.2 \cdot(i-1) \mathrm{m}, T R_{i}$ and $R E_{1, i}, T R_{i}$ and $R E_{3, i}$ are both: 


$$
L_{0}+0.6+0.2 \cdot(i-1)
$$

Where $i=1,2, \ldots, 10$.

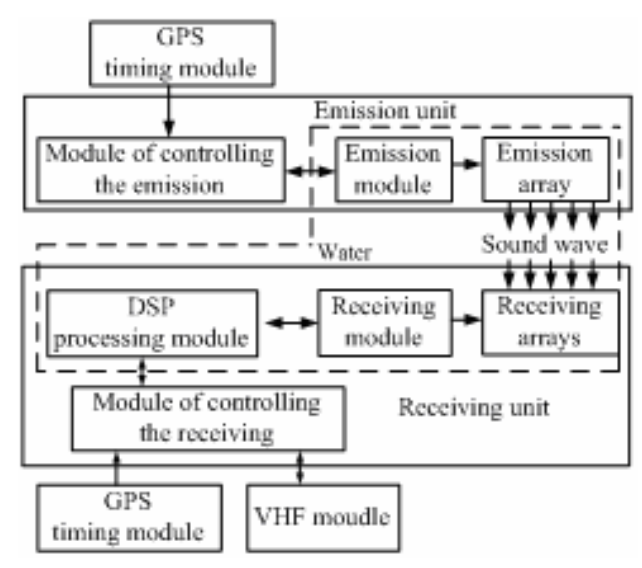

Fig.2: Scheme of system work

The monitoring system is mainly composed of the emission unit and the receiving unit. Moreover, the monitoring system consists of GPS timing module and VHF module. As shown in Fig.2, the part in the broken line is set up under the water, and through RS422 interface, the part under the water can communicate with the part above the water. When the normal monitoring is carried through, we can read out the precise time from the GPS timing module and then begin to emit and receive the sonar signal. The bandpass filter and the amplification circuit in the receiving module can filter the signal with noise and amplify the weak signal. The high speed $\mathrm{A} / \mathrm{D}$ converter in the data acquisition and processing module collects the signal and the DSP processor deals with the signal. Then the result is sent out to the watching center in the bank through the VHF module and is processed and displayed in the center. Thus the real-time monitoring on the siltation situation in the channel can be carried out.

\subsection{Signal acquistion}

As the system is working, the emission transducer $T R_{1}$ and all the receiving transducers are opened synchronously firstly. The emission unit emits the sine pulse sonar signal with $(200 \mathrm{kHz})$ frequency and $(1 \mathrm{~s})$ repetition frequency as well as $(0.2 \mathrm{~ms})$ pulse width. For the siltation of the channel is flat, so according to the direct wave features received from each receiving transducer, we can judge that if there exists siltation between the emission transducer $T R_{1}$ and the receiving transducer $R E_{1,1}$ or $R E_{2,1}$ or $R E_{3,1}$. And open other emission transducers in turn, the siltation situation of the section between the emission array and the receiving arrays can be monitored in real time.

For the adopted sonar signal is high frequency, in order to reduce the data acquisition amount, the way of delaying some time and setting time window (Tw) is applied. At first, applying GPS time module, the emission transducer $T R_{i}$ and the transducers of the receiving arrays $R E_{1}, R E_{2}, R E_{3}$ are opened synchronously. And data acquisition begins after delay the time $d t_{1}=1000 \cdot\left[L_{0}+0.6+0.2 \cdot(i-1)\right] / c \mathrm{~ms}$ and $d t_{2}=1000 \cdot\left[L_{0}+0.2 \cdot(i-1)\right] / c$ ms as well as $d t_{3}=1000 \cdot\left[L_{0}++0.6+0.2 \cdot(i-1)\right] / c \quad \mathrm{~ms}$ respectively $(i=1,2, \ldots, 10, \quad c$ is the acoustic velocity of the water area). Also the time can be as the start point of the Tw and the data acquisition length is 1.5 times of the pulse width (namely: $0.25 \mathrm{~ms}$ ).

For the acoustic velocity is affected by flood tide, ebb-tide, water temperature and salt, the time from the emission transducer to the receiving transducer varies constantly. Suppose that the maximum and minimum acoustic velocity of the water area is $C_{\max }$ and $C_{\min }$ respectively, and through measurement and calculation, $C_{\max }=1508 \mathrm{~m} / \mathrm{s}, C_{\min }=1493 \mathrm{~m} / \mathrm{s}$. In order to ensure that the set Tw contains the whole direct wave in any situation, the length of the Tw between the emission array and the receiving arrays is as the following in turn (the unit is milliseconds):

$$
\begin{aligned}
& \Delta t_{1}=1000 \cdot\left[L_{0}+0.6+0.2 \cdot(i-1)\right] \cdot\left(\frac{1}{c_{\min }}-\frac{1}{C_{\max }}\right)+0.25 \\
& \Delta t_{2}=1000 \cdot\left[L_{0}+0.2 \cdot(i-1)\right] \cdot\left(\frac{1}{C_{\min }}-\frac{1}{C_{\max }}\right)+0.25 \\
& \Delta t_{3}=1000 \cdot\left[L_{0}+0.6+0.2 \cdot(i-1)\right] \cdot\left(\frac{1}{c_{\min }}-\frac{1}{C_{\max }}\right)+0.25
\end{aligned}
$$

$$
i=1,2, \ldots, 10 \text {. }
$$

\subsection{Feature extraction}

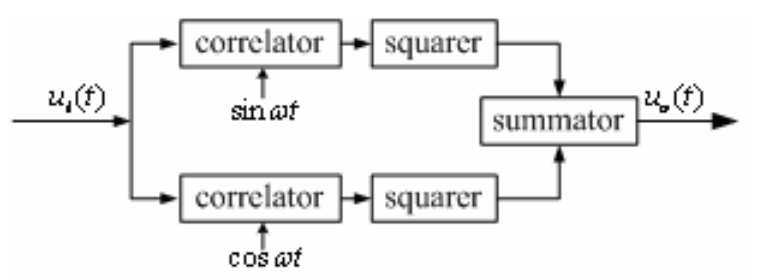

Fig.3: Structure of the random phase signal optimal receiver 
In turbid water area, the attenuation of the sonar signal is great and the ocean ambient noise is wide band random signal obeyed gauss distribution. In addition, in the Tw, the sonar signal is random phase signal (RPS). When extracting the features of the signal, in order to eliminate the influence on signal detection caused by the phase, RPS optimal receiver (as shown in Fig.3) can be adopted. In the process, firstly, use a slide time window (Stw, the window length is $0.2 \mathrm{~ms}$, the slide step is $10 \mu \mathrm{S})$ to select a length of original data as input signal $u_{i}(t)$ of the optimal receiver, and have cross-correlation operation of $u_{i}(t)$ and orthogonal reference signal ( $\sin \omega t, \cos \omega t$, with the same frequency as emission signal), then square and add the result, thus the output signal is obtained.

Through extracting the features of the received signal from transducers of the receiving array $R E_{2}$ as $T R_{1}$ emitting signal, the process of extracting the alluvial features can be explained. To the signal received from $R E_{2,1}$, set a time window $T w_{2,1}$ (from the formula (8), we can know the window length is $1.58 \mathrm{~ms})$. At first, slide time window Stw $w_{2,1}$ selects a section of original data from $T w_{2,1}$ as the input signal of the optimal receiver, and after being processed in the optimal receiver, the peak value $P v_{2,1-1}$ of the output signal is obtained.

Similarly, after the optimal receiver operates the signals received by $R E_{2,2}, R E_{2,3}, \ldots R E_{2,10}$, the maximum of the peak values of the output values are $P k_{2,2}, P k_{2,3}, \ldots, P k_{2,10}$ respectively and these values shows if the receiving transducers can receive the direct wave and the intensity of the direct wave. Furthermore, as whether the section between $T R_{1}$ and $R E_{1}$ is alluvial and the alluvial degree relates closely with the received signal, the values can be as the features of judging the siltation between $T R_{1}$ and $R E_{1}$. In this paper, support vector machines are introduced. In order to enhance the convergence rate and the judgement precision, the extracting values can be processed as following formula:

$$
x_{i}=\frac{2\left(P k_{2, i}-P k_{\min }\right)}{P k_{\max }-P k_{\min }}, i=1,2, \ldots, 10
$$

Where $P k_{\max }$ and $P k_{\min }$ are the maximum value the and minimum value respectively of $P k_{2,1}, P k_{2,2}, \ldots$, $P k_{2,10} \cdot\left(x_{1}, x_{2}, \ldots, x_{10}\right)$. And they are feature vector to judge the siltation between $T R_{1}$ and $R E_{1}$, namely it is the input sample of SVM.

The rest may be deduced by analogy similarly, the feature vectors can be obtained to judge the siltation between the other emission transducers and receiving transducers [8].

\section{Experimental results}

The recognition function is as formula (4), and the radial basis kernel function whose recognition veracity is high is selected [9]-[10].

Suppose that the sections are $S_{1}, S_{2}$ and $S_{3}$ respectively between the emission array and the receiving arrays $R E_{1}, R E_{2}, R E_{3}$ And the small sections between each emission transducer and its opposite receiving transducers in each section are $S_{1,1}$, $S_{1,2}, \ldots, S_{1,10} ; S_{2,1}, S_{2,2}, \ldots, S_{2,10} ; S_{3,1}, S_{3,2}, \ldots$, $S_{3,10}$. And to each small section, choose the extracted signal features and the siltation situation (60 groups of data) during the period from Nov. 20, 2006 to Apr. 5, 2007 in all kinds of weather as the samples. The siltation situation was measured by the high precision single frequency echo sounder Bathy-500MF produced in ODEC of America. Among the samples, 40 groups are used to trained, 20 groups are used to test. In order to prove the efficiency of SVM method to recognize the siltation, the traditional recognition methods BP neural network and RBF neural network are introduced. The siltation recognition results of the three methods are shown in the following table:

\begin{tabular}{|c|c|c|c|}
\hline Section & SVM & BP & RBF \\
\hline $\mathrm{S} 1,1$ & $91.60 \%$ & $64.20 \%$ & $69.40 \%$ \\
\hline $\mathrm{S} 1,2$ & $92.60 \%$ & $66.20 \%$ & $70.60 \%$ \\
\hline $\mathrm{S} 1,3$ & $96.90 \%$ & $67.30 \%$ & $78.00 \%$ \\
\hline $\mathrm{S} 1,4$ & $93.50 \%$ & $69.20 \%$ & $73.60 \%$ \\
\hline $\mathrm{S} 1,5$ & $95.00 \%$ & $60.70 \%$ & $72.30 \%$ \\
\hline $\mathrm{S} 1,6$ & $94.40 \%$ & $66.30 \%$ & $77.10 \%$ \\
\hline $\mathrm{S} 1,7$ & $91.70 \%$ & $64.70 \%$ & $75.40 \%$ \\
\hline $\mathrm{S} 1,8$ & $92.50 \%$ & $61.90 \%$ & $77.80 \%$ \\
\hline $\mathrm{S} 1,9$ & $97.90 \%$ & $64.60 \%$ & $70.30 \%$ \\
\hline $\mathrm{S} 1,10$ & $92.70 \%$ & $69.20 \%$ & $72.60 \%$ \\
\hline S2,1 & $91.20 \%$ & $65.90 \%$ & $76.60 \%$ \\
\hline $\mathrm{S} 2,2$ & $93.30 \%$ & $62.00 \%$ & $67.10 \%$ \\
\hline $\mathrm{S} 2,3$ & $93.60 \%$ & $68.70 \%$ & $68.80 \%$ \\
\hline $\mathrm{S} 2,4$ & $94.10 \%$ & $62.40 \%$ & $79.10 \%$ \\
\hline $\mathrm{S} 2,5$ & $92.90 \%$ & $64.10 \%$ & $75.30 \%$ \\
\hline
\end{tabular}




\begin{tabular}{|c|c|c|c|}
\hline $\mathrm{S} 2,6$ & $92.00 \%$ & $69.00 \%$ & $76.40 \%$ \\
\hline $\mathrm{S} 2,7$ & $91.50 \%$ & $67.60 \%$ & $79.50 \%$ \\
\hline $\mathrm{S} 2,8$ & $92.40 \%$ & $65.70 \%$ & $70.90 \%$ \\
\hline $\mathrm{S} 2,9$ & $96.50 \%$ & $68.10 \%$ & $71.00 \%$ \\
\hline $\mathrm{S} 2,10$ & $90.90 \%$ & $69.80 \%$ & $74.50 \%$ \\
\hline $\mathrm{S} 3,1$ & $96.40 \%$ & $69.10 \%$ & $73.90 \%$ \\
\hline $\mathrm{S} 3,2$ & $91.90 \%$ & $60.50 \%$ & $72.10 \%$ \\
\hline $\mathrm{S} 3,3$ & $96.70 \%$ & $68.20 \%$ & $76.60 \%$ \\
\hline $\mathrm{S} 3,4$ & $93.80 \%$ & $64.80 \%$ & $70.30 \%$ \\
\hline $\mathrm{S} 3,5$ & $92.70 \%$ & $65.30 \%$ & $77.30 \%$ \\
\hline $\mathrm{S} 3,6$ & $93.10 \%$ & $62.50 \%$ & $70.10 \%$ \\
\hline $\mathrm{S} 3,7$ & $94.60 \%$ & $61.70 \%$ & $69.70 \%$ \\
\hline $\mathrm{S} 3,8$ & $96.80 \%$ & $64.40 \%$ & $73.30 \%$ \\
\hline $\mathrm{S} 3,9$ & $90.80 \%$ & $70.50 \%$ & $76.70 \%$ \\
\hline $\mathrm{S} 3,10$ & $97.50 \%$ & $68.30 \%$ & $75.60 \%$ \\
\hline
\end{tabular}

Tab.1: Siltation recognition results of three methods.

The experimental results show that SVM is a practical machine learning approach to recognize the channel siltation. Compared to other traditional learning approaches, the approach of SVM has good judgement effect, high recognition precision and good generalization, especially, it is suitable to small sample problem and can not cause over-trainning problem.

\section{Conclusions}

In this paper, a new method to monitor the siltation situation was developed, which just needs to set up some transducer arrays on the sides of the channel and the channel siltation issue is transformed to a pattern recognition question. Applying the constructed SVM, the channel siltation of one port was recognized correctly. The results show that the approach to recognize channel siltation is practical.

Compare with traditional approaches to recognize the channel siltation such as BP neural network and RBF neural network, SVM approach has high recognition precision $(>90 \%)$ and better generalization. So it can be used in engineering.

\section{References}

[1] J.D. Milliman, R. H. Meade, World-wide delivery of river sediment to the oceans. Journal of Geology, 91:1-21, 1983.

[2] M.A. Prins, Postma. G., Controls on terrigenous sediment supply to the Arabian Sea during the late Quaternary: the Indus Fan. Marine Geology, 169:327-349, 2000.

[3] H.B. Zhao, X.S. Xu, Perspective of development of multi-beam swath techniques.
Journal of Harbin Engineering University, 22: 41-45, 2001.

[4] J.H. Satrino, Wide swath bathymetry from multibeam systems. IEEE, 3(16):733-736, 1991.

[5] G. Nelson, V. Durdle, A support vector machines classifier to assess the severity of idiopathic scoliosis from surface topography. IEEE Trans. on Information Technology in Biomedicine, 1:124-126, 2006.

[6] V.N. Vapnik, An overview of statistical learning theory. IEEE Trans. Neural Netw, 5:988-999, 1999.

[7] K.R. Muller, S. Mika, G. Ratsch, An introduction to kernel-based learning algorithms. IEEE Trans. Neural Netw, 12:181-201, 2001.

[8] M. Lehrman, A. B. Rechester, Symbolic analysis of chaotic signals and turbulent fluctuations. Physical Review Letters, 78:54-57, 1997.

[9] I. Buciu, C. Kotropoulos, Demonstrating the stability of support vector machines for classification. Signal Process, 86:2364-2380, 2006.

[10] H.C. Kim, S. Pang, Constructing support vector machine ensemble. Pattern Recognition, 36:2757-2767, 2003. 